\title{
Model-Based Geostatistics for Prevalence Mapping in Low-Resource Settings
}

\author{
Peter J Diggle and Emanuele Giorgi \\ (Lancaster Medical School, Lancaster University)
}

December 18, 2015

\begin{abstract}
In low-resource settings, prevalence mapping relies on empirical prevalence data from a finite, often spatially sparse, set of surveys of communities within the region of interest, possibly supplemented by remotely sensed images that can act as proxies for environmental risk factors. A standard geostatistical model for data of this kind is a generalized linear mixed model with binomial error distribution, logistic link and a combination of explanatory variables and a Gaussian spatial stochastic process in the linear predictor. In this paper, we first review statistical methods and software associated with this standard model, then consider several methodological extensions whose development has been motivated by the requirements of specific applications. These include: methods for combining randomised survey data with data from non-randomised, and therefore potentially biased, surveys; spatio-temporal extensions; spatially structured zero-inflation. Throughout, we illustrate the methods with disease mapping applications that have arisen through our involvement with a range of African public health programmes.
\end{abstract}

Keywords: geostatistics; multiple surveys; prevalence; spatio-temporal models; zeroinflation. 


\section{Introduction}

The term "geostatistics" is typically used as a convenient shorthand for statistical models and methods associated with analysing spatially discrete data relating to an unobserved spatially continuous phenomenon. The name derives from its origins in the South African mining industry (Krige, 1951) and its subsequent development by the late Georges Matheron and colleagues in L'École des Mines, Fontainebleau, France (Chilés \& Delfiner, 2012). Geostatistical methodology has since been applied in a wide range of scientific contexts, and is now widely accepted as one of three main branches of spatial statistics (Cressie, 1993). The descriptive phrase "model-based geostatistics" was coined by Diggle, Tawn \& Moyeed (1998) to mean the embedding of geostatistics within the general framework of statistical modelling and likelihood-based inference as applied to geostatistical problems. In contrast, "classical" Fontainebleau-style geostatistics has its own terminology and self-contained methodology, developed largely independently of the statistical mainstream.

Whether tackled through the model-based or classical approach, a typical feature of most geostatistical problems is a focus on prediction rather than on parameter estimation. The canonical geostatistical problem, expressed in the language of model-based geostatistics, is the following. Data $\left\{\left(y_{i}, x_{i}\right): i=1, \ldots, n\right\}$ are realised values of random variables $Y_{i}$ associated with pre-specified locations $x_{i} \in A \subset \mathbb{R}^{2}$. The $Y_{i}$ are assumed to be statistically dependent on an unobserved stochastic process, $\mathcal{S}=\left\{S(x): x \in \mathbb{R}^{2}\right\}$, as expressed through a statistical model $[S, Y]=[S][Y \mid S]$, where [.] means "the distribution of," $Y=\left(Y_{1}, \ldots, Y_{n}\right)$ and $S=$ $\left\{S\left(x_{1}\right), \ldots, S\left(x_{n}\right)\right\}$. What can be said about the realisation of $\mathcal{S}$ ? The formal model-based solution is the conditional distribution, $[\mathcal{S} \mid Y]=[\mathcal{S} \mid S][S \mid Y]$, where $[S \mid Y]$ follows as a direct application of Bayes' theorem,

$$
[S \mid Y]=[S][Y \mid S] / \int[S][Y \mid S] d S
$$


By far the most tractable case is the linear Gaussian model, for which $\mathcal{S}$ is a Gaussian process and the $Y_{i}$ given $S$ are conditionally independent, $Y_{i} \mid S \sim \mathrm{N}\left(S\left(x_{i}\right), \tau^{2}\right)$. It follows that the marginal distribution of $Y$, the conditional distribution $[S \mid Y]$ and the conditional distribution $[\mathcal{S} \mid S]$ are all multivariate Normal.

Note that in the above formulation, no model is specified for the $x_{i}$. We return to this point in the discussion, but in the meantime the implicit assumption is that the $x_{i}$ are prespecified as part of the study-design or are located according to a process that is stochastically independent of $S$. If $X=\left(x_{1}, \ldots, x_{n}\right)$ is stochastic, a complete factorisation of the model for the data is $[S, X, Y]=[S][X \mid S][Y \mid X, S]$. Then, if $[X \mid S]=[X]$ and the properties of $[X]$ are not of interest, it is legitimate to condition on $[X]$ and so recover the previous formulation, $[S, Y]=[S][Y \mid S]$

Diggle, Moraga, Rowlingson \& Taylor (2013) argue that the geostatistical label should be applied more generally to scientific problems that involve predictive inference about an unobserved spatial phenomenon $S(x)$ using any form of incomplete information. This includes, for example, predictive inference for the intensity of a Cox process (Cox, 1955), and inference when $X$ is both stochastic and dependent on $S$.

In this paper, we restrict our substantive scope to the problem of analysing data from spatially referenced prevalence surveys. We also focus on prevalence mapping in low-resource countries where registry data are lacking. We argue that in low-resource settings the sparsity of the available data justifies a more strongly model-based approach than would be appropriate if accurate registries were available.

\section{The standard geostatistical model for prevalence data}

In its most basic form, a prevalence survey consists of visiting communities at locations $x_{i}$ : $i=1, \ldots, n$ distributed over a region of interest $A$ and, in each community, sampling $m_{i}$ 
individuals and recording whether each tests positive or negative for the disease of interest. If $p(x)$ denotes prevalence at location $x$, the standard sampling model for the resulting data is binomial, $Y_{i} \sim \operatorname{Bin}\left(m_{i}, p\left(x_{i}\right)\right)$ for $i=1, \ldots, n$. Linkage of the $p\left(x_{i}\right)$ at different locations is usually desirable, and is essential if we wish to make inferences about $p(x)$ at unsampled locations $x$.

The simplest extension to the basic model is a binary regression model, for example a logistic regression model of the form

$$
\log \left[p\left(x_{i}\right) /\left\{1-p\left(x_{i}\right)\right\}\right]=d\left(x_{i}\right)^{\prime} \beta
$$

where $d\left(x_{i}\right)$ is a vector of explanatory variables associated with the location $x_{i}$. This assumes that the value of $d(x)$ is available not only at the data-locations $x_{i}$ but also at any other location $x$ that is of interest. When extra-binomial variation is present, two further extensions are possible. Firstly, a standard mixed effects model adds a random effect to the right-handside of (1), to give

$$
\log \left[p\left(x_{i}\right) /\left\{1-p\left(x_{i}\right)\right\}\right]=d\left(x_{i}\right)^{\prime} \beta+Z_{i}
$$

where the $Z_{i}$ are independent $\mathrm{N}\left(0, \tau^{2}\right)$ variates. Secondly, if the context suggests that covariateadjusted prevalence should vary smoothly over the region of interest, we can add a spatially correlated random effect, to give

$$
\log \left[p\left(x_{i}\right) /\left\{1-p\left(x_{i}\right)\right\}\right]=d\left(x_{i}\right)^{\prime} \beta+S\left(x_{i}\right)+Z_{i}
$$

where $\mathcal{S}=\left\{S(x): x \in \mathbb{R}^{2}\right\}$ is a Gaussian process with mean zero, variance $\sigma^{2}$ and correlation function $\rho\left(x, x^{\prime}\right)=\operatorname{Corr}\left\{S(x), S\left(x^{\prime}\right)\right\}$. We shall assume that the process $\mathcal{S}$ is stationary and isotropic, hence $\operatorname{Corr}\left\{S(x), S\left(x^{\prime}\right)\right\}=\rho\left(\left\|x-x^{\prime}\right\|\right)$, where $\|\cdot\|$ denotes the Euclidean distance. Amongst the various parametric families that have been proposed for $\rho(u)$, Stein (1999) ad- 
vocates the use of the Matérn (1986) correlation function, given by

$$
\rho(u ; \phi, \kappa)=\left\{2^{k-1} \Gamma(\kappa)\right\}^{-1}\left(\left\|x-x^{\prime}\right\| / \phi\right)^{\kappa} \mathcal{K}_{\kappa}\left(\left\|x-x^{\prime}\right\| / \phi\right), u>0,
$$

where $\phi>0$ is a scale parameter and $\mathcal{K}_{\kappa}(\cdot)$ is the modified Bessel function of the second kind of order $\kappa>0$. The shape parameter $\kappa$ determines the smoothness of $S(x)$, in the sense that $S(x)$ is $\lceil\kappa\rceil-1$ times mean-square differentiable, with $\lceil\kappa\rceil$ denoting the smallest integer greater than or equal to $\kappa$. The special case of $\kappa=1 / 2$ corresponds to the exponential correlation function which generates mean-square continuous processes $S(x)$. The tangible interpretation of the $\kappa$ parameter is advantageous because Zhang (2004) has shown that the parameters $\sigma^{2}, \phi$ and $\kappa$ can not all be consistently estimated under in-fill asymptotics. In practice this translates to $\kappa$ often being poorly identified. Our strategy is therefore to use the Matérn family, fixing $\kappa$ at a plausible value consistent with initial exploratory analysis of the variogram based on the residuals of a non-spatial logistic regression model. Note that the Matérn family includes the non-stationary de Wijs process (de Wijs, 1951, 1953) as a limiting case when $\kappa \rightarrow 0$ (Besag and Mondal, 2005).

The initial focus of inference within model (2) is the unobserved surface $p(x)$ or specific properties thereof. In general, we call $T=\mathcal{T}(\mathcal{S})$ a target for predictive inference. For example, we may wish to delineate sub-regions of $A$ where $p(x)$ is likely to exceed a policy intervention threshold, in which case the target is $T=\{x: p(x)>c\}$ for pre-specified $c$, and the required output from the analysis is the predictive distribution of the random set $T$.

Equation (2) defines what we shall call the standard geostatistical prevalence sampling model. Various approaches to fitting this model to geostatistical data have been suggested in the literature. Diggle, Tawn \& Moyeed (1998) used Bayesian inference for parameter estimation and prediction, implemented by an MCMC algorithm. Rue, Martino \& Chopin (2009) used integrated nested Laplace approximation (INLA) methods. INLA methods can be used more generally for fitting generalized linear mixed models with a residual Markov dependence struc- 
ture. Also, Lindgren, Rue \& Lindstrm (2011) has shown that random field models can be expressed as solutions to continuous domain stochastic partial differential equations (SPDEs). This gives an explicit link between the parameters of each SPDE and the elements of the corresponding precision matrix in a spatially discrete basis-function representation. The practical consequence of this is that INLA can be used for approximate inference on spatially continuous Gaussian random fields using computationally fast methods based on spatially discrete Markov random fields. The INLA methodology and its associated software yield accurate and computationally fast approximations to the marginal posterior distributions of model parameters and to the marginal predictive distribution of $S(x)$ at each element of a set of locations $x$, but not to their joint predictive distribution. This limits INLA's applicability to point-wise targets $T$, whereas in many applications the targets $T$ involves the complete process $\mathcal{S}$ or, in practice, its restriction to a fine grid of locations to cover the region of interest; examples include area-wide summaries of $S(x)$, such as its spatial average or the proportion over a specified threshold. In the application of Section 5, we give an example where the target is the village-level average of malaria prevalence.

Giorgi \& Diggle (2014) provide an R package for Monte Carlo maximum likelihood estimation and plug-in prediction with an option to use a low-rank approximation to $\mathcal{S}$ for faster computation with large data-sets. The low-rank method approximates $\mathcal{S}$ by $\mathcal{S}^{*}$, where

$$
S^{*}(x)=\sum_{k=1}^{r} f\left(x-x_{k}\right) V_{k}
$$

In (4), the $V_{k}$ are independent $\mathrm{N}\left(0, \tau^{2}\right)$ variates associated with a pre-specified set of locations $x_{k}$ and $f(x)$ is a prescribed function, typically monotone non-increasing in $\|x\|$. The covariance function of $\mathcal{S}^{*}$ is

$$
\operatorname{Cov}\left\{S^{*}(x), S^{*}\left(x^{\prime}\right)\right\}=\tau^{2} \sum_{k=1}^{r} f\left(x-x_{k}\right) f\left(x^{\prime}-x_{k}\right),
$$

Low-rank specifications have been proposed as models in their right; see, for example, Higdon (1998, 2002). We consider them as approximations to a limiting, full-rank process. Taking 
the $x_{k}$ in (4) as the points of an increasingly fine regular lattice and scaling the function $f(\cdot)$ commensurate with the lattice spacing gives a limiting, full-rank process with covariance function

$$
\operatorname{Cov}\left\{S^{*}(x), S^{*}\left(x^{\prime}\right)\right\}=\tau^{2} \int_{\mathbb{R}^{2}} f(x-u) f\left(x^{\prime}-u\right) d u .
$$

From this perspective, the summation in (5) represents a quadrature approximation to the integral in (6). As the scale of the spatial correlation increases, a smaller number of $x_{k}$ points is required for an adequate approximation. In practice, we start with a computationally convenient value for $r$ which we then increase until the spatial predictions for a specific target stabilise; Giorgi \& Diggle (2014) give an example on a simulated data-set.

Gotway \& Stroup (1997) suggest using generalized estimating equations (Liang \& Zeger, 1986) when scientific interest is focused on the regression parameters rather than on prediction of $\mathcal{S}$. However, in this approach the implicit target for inference is not the parameter vector $\beta$ that appears in (2), but rather the marginal regression parameter vector, $\beta^{*}$ say. The elements of $\beta^{*}$ are smaller in absolute value than those of $\beta$ by an amount that depends on $\tau^{2}, \sigma^{2}$ and $\rho(u)$.

Diggle et al. (2007) use the standard model (2), but without the mutually independent random effects $Z_{i}$, to construct predictive maps of the prevalence of Loa loa, a parasitic infection of the eye, in an area of equatorial west Africa covering Cameroon and parts of its neighbouring countries. Following Thomson et al. (2004) they include two remotely sensed covariates, height above sea-level and the Normalised Digital Vegetation Index (NDVI), as proxies for the ability of the disease vector, a particular species of Chrysops fly, to breed at each location. As described in Thomson et al. (2004) and Diggle et al. (2007), Loa loa prevalence mapping plays an important role in the implementation of a multi-national prophylactic mass-treatment programme for the control of onchocerciasis (river blindness), the African Programme for Onchocerciasis Control, APOC (WHO, 2012), following the recognition that a generally safe filaricide medication, Ivermectin, could produce severe, occasionally fatal, adverse reactions 


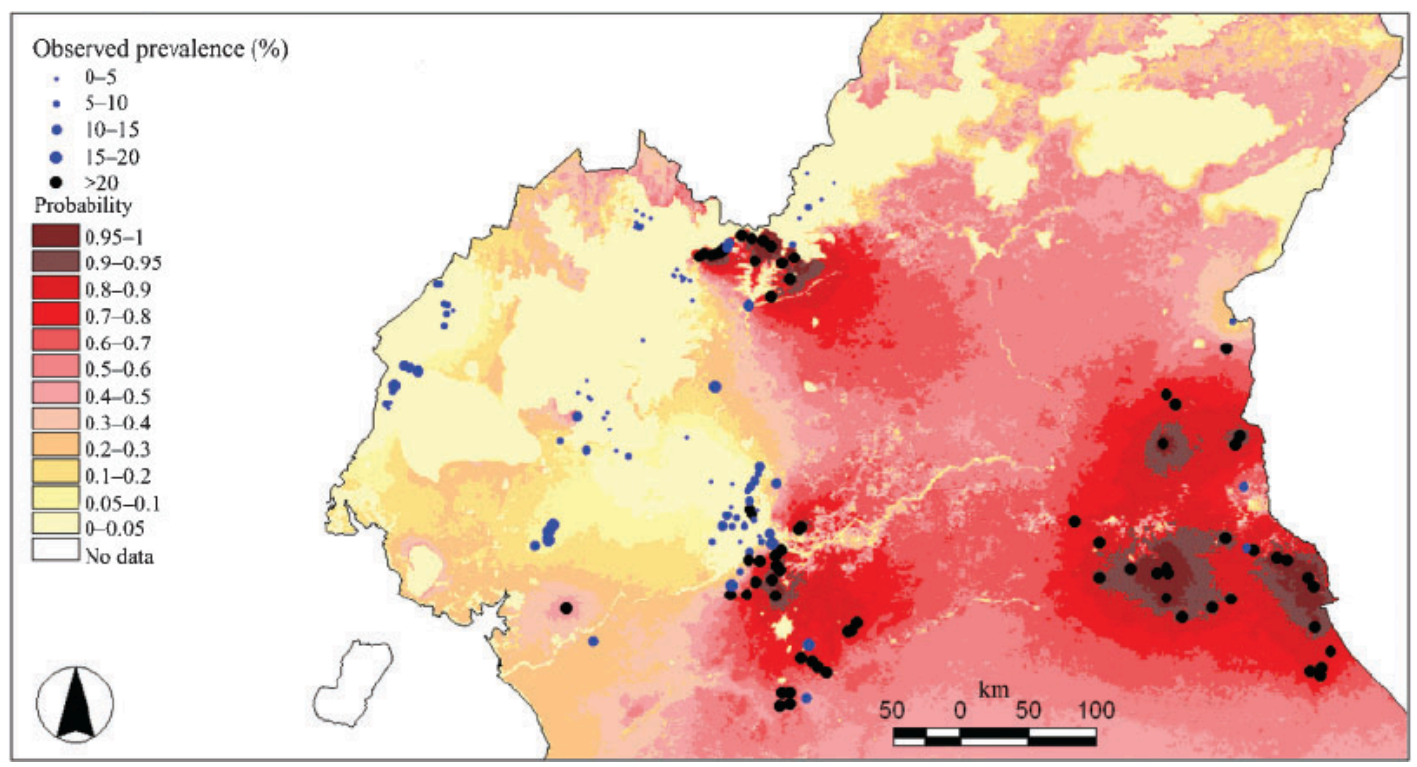

Figure 1: Predictive probability map of Loa loa prevalence in Cameroon and surrounding areas (adapted from Diggle et al. (2007)). Empirical prevalences at surveyed locations are indicated by size and colour coded dots.

in people heavily co-infected with onchocerciasis and Loa loa parasites. As a result, APOC adopted the policy that in areas where Loa loa prevalence was greater than 20\%, precautionary measures should be taken before local administration of Ivermectin.

Diggle et al. (2007) mapped the minimum mean square error point predictor, $\mathrm{E}[p(x) \mid y]$ but also argued that a more useful quantity was the point-wise predictive probability, $q(x)$ say, that $p(x)$ exceeded 0.2 , in line with APOC's precautionary policy. In addition to addressing directly the relevant practical problem, a map of $q(x)$ conveys the uncertainty associated with the resulting predictions. This map, here reproduced as Figure 1, identifies large areas that almost certainly do and do not meet the policy-intervention criterion, but also delineates large areas where the only honest answer is "don't know," indicating the need for further investigation or, if practicalities dictate, taking an informed risk.

Other prevalence mapping applications of model-based geostatistics include: Claridge et al. (2012) on liver fluke and bovine tuberculosis in the UK cattle herd; Clements et al. (2006) on schistosomiasis in Tanzania; Diggle et al. (2002) on childhood malaria in the Gambia; 
Gemperli et al. (2004) on infant mortality in Mali; Gething et al. (2012) on the world-wide distribution of Plasmodium vivax; Hay et al. (2009) on the world-wide distribution of Plasmodium falciparium; Kleinschmidt et al. (2001) on malaria incidence in Kwazuku Natal, South Africa; Kleinschmidt et al. (2007) on HIV in South Africa; Soares Magalhaes \& Clements (2011) on anemia in preschool-aged children in West Africa; Raso et al. (2005) on schistosomiasis in Côte D'Ivoire; Pullan et al. (2011) on soil-transmitted infections in Kenya; Zouré et al. (2014) on river blindness in the 20 participating countries of the African Programme for Onchocerciasis control.

\section{Computational details}

In each of the applications to be described in Section 4 to 6, we use the Monte Carlo maximum likelihood (MCML) method for parameter estimation. This fitting procedure can be used under a very general modelling framework. Let $W_{i}$ for $i=1, \ldots, n$ denote a set of random effects associated with $Y_{i}$, following a joint multivariate Normal distribution with mean $\mu$ and covariance matrix $\Sigma$. Assume that $Y_{i}$ conditionally on $W_{i}$ are mutually independent random variables with distributions $f\left(\cdot \mid W_{i}\right)$. The likelihood function for the vector of model parameters $\theta$ is given by

$$
\begin{aligned}
L(\theta) & =\int_{\mathbb{R}^{\operatorname{dim}(\mathrm{W})}} g(W, y ; \theta) d W \\
& =\int_{\mathbb{R}^{\operatorname{dim}(\mathrm{W})}} N(W ; \mu, \Sigma) \prod_{i}^{n} f\left(y_{i} \mid W_{i}\right) d W
\end{aligned}
$$

where $\operatorname{dim}(W)$ denotes the dimension of $W$. Note, for example, that in the model used in Section 6.1, the random effect associated with village $i$ is a bivariate random variable, $W_{i}=\left\{S\left(x_{i}\right)+Z_{i}, T\left(x_{i}\right)\right\}$, hence $\operatorname{dim}(W)=2 n$ with $f\left(\cdot \mid W_{i}\right)$ given by (14). Monte Carlo methods are then used in order to approximate the above intractable integral using importance sampling. As discussed in Giorgi \& Diggle (2014), a convenient choice for the importance 
sampling distribution is $g\left(W, y ; \theta_{0}\right)$ for some fixed $\theta_{0}$, which can be iteratively updated. With this choice, a Markov chain Monte Carlo (MCMC) algorithm is then required for simulation of $W_{i}$ conditionally on $y_{i}$ under $\theta_{0}$. We use a Langevin-Hastings algorithm that updates the transformed vector of random effects $\hat{\Sigma}^{-1 / 2}(W-\hat{W})$, where $\hat{W}$ and $\hat{\Sigma}$ are the mode and the inverse of the negative Hessian at $\hat{W}$ of $g\left(W, y ; \theta_{0}\right)$.

\section{Combining information from multiple surveys}

In order to obtain good geographical coverage of the population of interest, it is often necessary to combine information from multiple prevalence surveys. However, understanding the limitations of the sampling design adopted in each survey is crucial in order to draw valid inferences from a joint analysis of the data. In particular, non-randomized "convenience" surveys in which data are gathered opportunistically, for example at schools, markets or hospital clinics, may reach an unrepresentative sub-population or be biased in other ways. Nonetheless, convenience samples represent a tempting, low-cost alternative to random samples. A combined analysis of data from randomised and convenience samples that estimates and adjusts for bias can be more efficient than an analysis that considers only the data from randomised surveys. In a non-spatial context, Hedt \& Pagano (2011) propose a hybrid estimator of prevalence that supplements information from random samples with convenience samples, and show that this leads to more accurate prevalence estimates than those available from using only the data from randomised surveys.

Giorgi et al. (2015) develop a multivariate generalized linear geostatistical model to account for data-quality variation amongst spatially referenced prevalence surveys. They assume that at least one of the available surveys is a "gold-standard" that delivers unbiased prevalence estimates and for which the standard model (2) is appropriate. Bias in a "non gold-standard" survey is then modelled using covariate information together with an additional, zero-mean 
stationary Gaussian process $\mathcal{B}=\left\{B(x): x \in \mathbb{R}^{2}\right\}$. The resulting model for a non-randomised survey is

$$
\log \left[p\left(x_{i}\right) /\left\{1-p\left(x_{i}\right)\right\}\right]=d\left(x_{i}\right)^{\prime} \beta+S\left(x_{i}\right)+Z_{i}+\left\{d\left(x_{i}\right)^{\prime} \delta+B\left(x_{i}\right)\right\}
$$

Data from both the randomised and the non-randomised survey then contribute to inference on the predictive target, $d(x)^{\prime} \beta+S(x)$.

\subsection{Application: using school and community surveys to estimate malaria prevalence in Nyanza Province, Kenya}

We now show an application to malaria prevalence data from a community survey and a school survey conducted in July 2010 in Rachuonyo South and Kisii Central Districts, Nyanza Province, Kenya. In the community survey, all residents above the age of 6 months were eligible for inclusion. A finger-prick blood sample was collected on each participant and examined for presence/absence of malaria parasites by a rapid diagnostic test (RDT).

In the school survey, 46 out of 122 schools with at least 100 pupils were randomly selected using an iterative process to limit the probability of selecting school with overlapping catchment areas. All eligible children in attendance were included. In the community survey, residential compounds lying within 600 meters of each school were randomly sampled and all eligible residents in each sampled compound examined by the RDT. The design of the community survey delivers an unbiased sample of residents from the catchment area of each school, whereas the school survey is potentially biased by a plausible association between a child's health status and their attendance at school. More details on the survey procedures can be found in Stevenson et al. (2013).

In our analysis, we extracted information on sampled individuals between the ages of 6 and 25 years in both surveys, as some adults have taken advantage of the introduction of free primary education in Kenya. The community survey included 1430 individuals distributed over 740 
compounds whilst the school survey included 4852 pupils distributed over 3791 compounds, i.e. averages per compound of approximately 1.9 and 1.3 people, respectively. Figure 2 shows the locations of the sampled compounds from both surveys.

For our joint analysis of the data from both surveys, we used exponential correlation functions for both $S(x)$ and $B(x)$, with $\phi$ and $\psi$ denoting the respective scale parameters. We parameterise the respective variances of $S(x), B(x)$ and $Z_{i}$ as $\sigma^{2}, \nu^{2} \sigma^{2}$ and $\omega^{2} \sigma^{2}$.

For selection of significant explanatory variables we used ordinary logistic regression, retaining variables with nominal $p$-values smaller than $5 \%$. Table 1 gives the final set of explanatory variables included in the geostatistical model. The "District" indicator variable accounts for a known higher level of malaria risk in Rachuonoyo district. Socio-economic status (SES) is an indicator of household wealth taking discrete values from 1 (poor) to 5 (wealthy).

Table 2 reports Monte Carlo maximum likelihood estimates and 95\% confidence intervals for the model parameters. The $\beta$-parameters reflect the district effect mentioned above as well as confirming a lower risk of malaria associated with higher scores of SES and greater age. The negative estimate of $\delta_{0}$ and its associated confidence interval indicate a significantly lower malaria prevalence in individuals attending school than in the community at large. The positive estimate and associated confidence interval for $\delta_{1}$ indicate that for individuals attending school, the negative effect of age is less strong than in the community. Figure 3(a) shows point-wise predictions of $B^{*}(x)=\exp \{B(x)\}$, which represents the unexplained multiplicative spatial bias in the school survey for the odds of malaria at location $x$. Figure 3(b) maps the predictive probability, $r(x)$ say, that $B^{*}(x)$ lies outside the interval $(0.9,1.1)$,

$$
r(x)=1-P\left(0.9<B^{*}(x)<1.1 \mid y\right) .
$$

The lowest value of $r(x)$ is about $87 \%$, indicating the presence of non-negligible spatially structured bias throughout the study area. The joint analysis of the data from both surveys allows us to remove the bias and so obtain more accurate predictions for $S(x)$ than would be 
Table 1: Explanatory variables used in the analysis of the Kenya malaria prevalence data.

\begin{tabular}{ll}
\hline & Term \\
\hline$\beta_{0}$ & Intercept \\
$\beta_{1}$ & Age in years \\
$\beta_{2}$ & District (=1 if "Rachuonyo"; =0 otherwise) \\
$\beta_{3}$ & Socio-economic status (score from 1 to 5 ) \\
$\delta_{0}$ & Survey indicator, 1 if "school," 0 if "community" (bias term) \\
$\delta_{1}$ & Age in years (bias term) \\
\hline
\end{tabular}

Table 2: Monte Carlo maximum likelihood estimates and corresponding 95\% confidence intervals for the model fitted to the Kenya malaria prevalence data

\begin{tabular}{ccc}
\hline & Estimate & $95 \%$ Confidence interval \\
\hline$\beta_{0}$ & -1.412 & $(-2.303,-0.521)$ \\
$\beta_{1}$ & -0.141 & $(-0.174,-0.109)$ \\
$\beta_{2}$ & 2.006 & $(1.228,2.785)$ \\
$\beta_{3}$ & -0.121 & $(-0.169,-0.072)$ \\
$\delta_{0}$ & -0.761 & $(-1.354,-0.167)$ \\
$\delta_{1}$ & 0.094 & $(0.046,0.142)$ \\
$\log \left(\sigma^{2}\right)$ & 0.519 & $(0.048,0.990)$ \\
$\log \left(\nu^{2}\right)$ & -1.264 & $(-1.738,-0.790)$ \\
$\log (\phi)$ & -3.574 & $(-4.083,-3.064)$ \\
$\log \left(\omega^{2}\right)$ & -1.408 & $(-2.267,-0.550)$ \\
$\log (\psi)$ & -3.366 & $(-4.178,-2.553)$ \\
\hline
\end{tabular}

obtained using only the data from the community survey. Figure 4(a) shows a scatter plot of the standard errors for $S(x)$ obtained from the joint model for the school and community surveys and from the model fitted to the community data only. Figure 4(b) shows that locations for which the joint analysis produces a larger standard errors for $S(x)$ correspond to areas where no observations were made. 


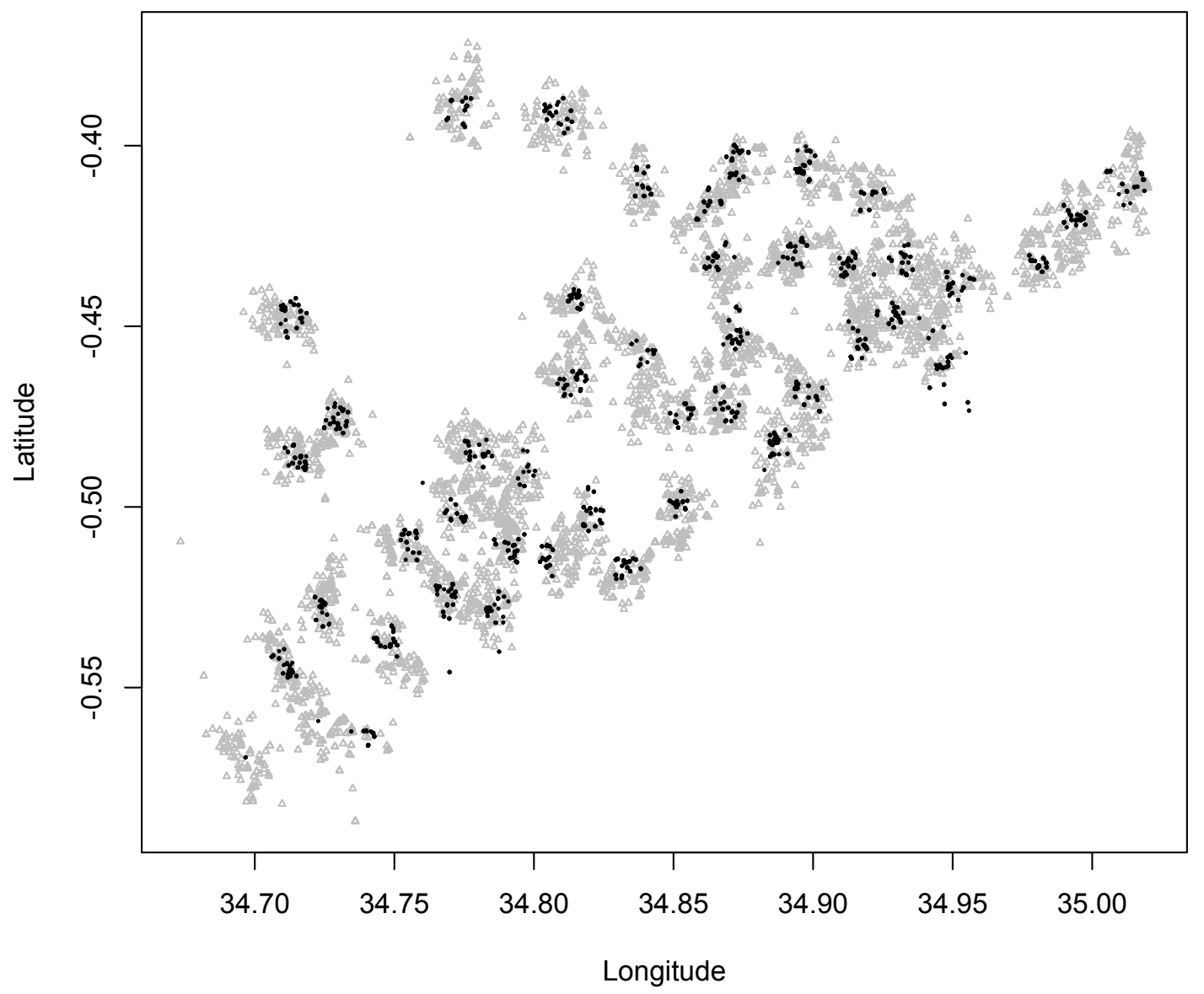

Figure 2: Geographical coordinates of the sampled compounds in the community (black dots) and school (grey traingles) surveys. 
(a)

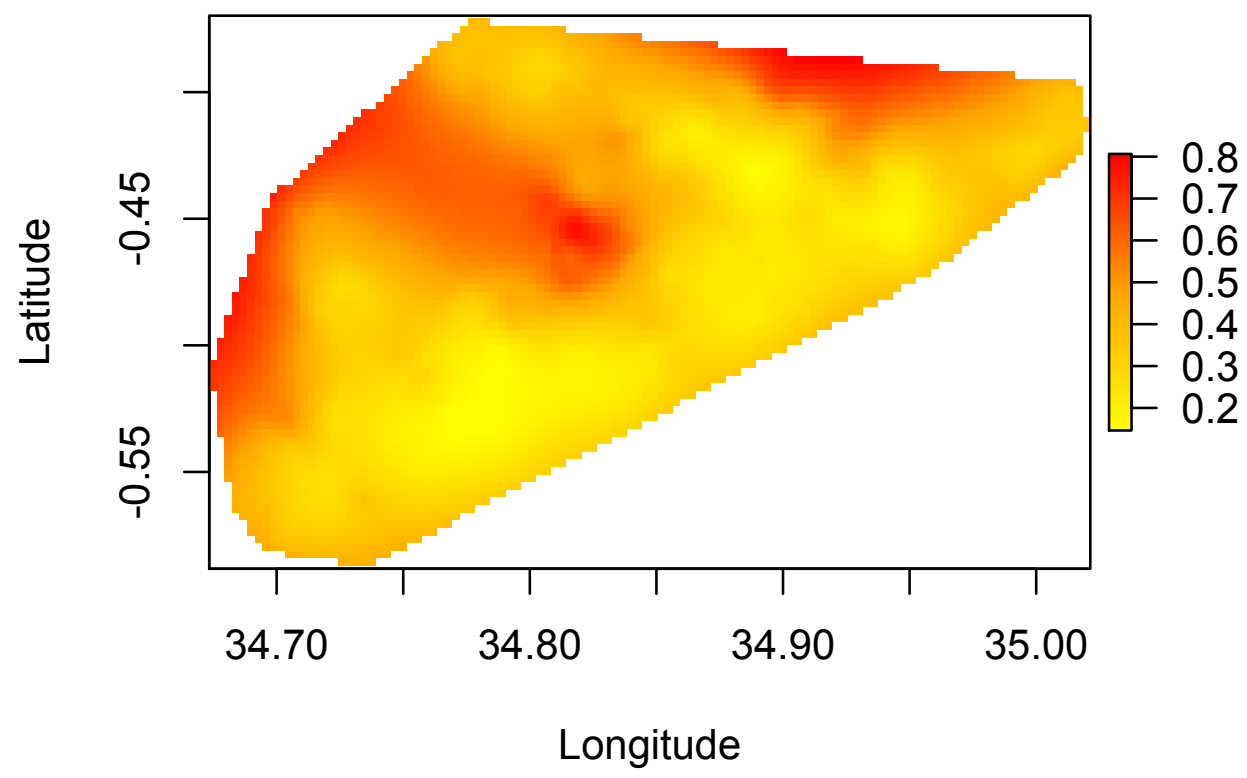

(b)

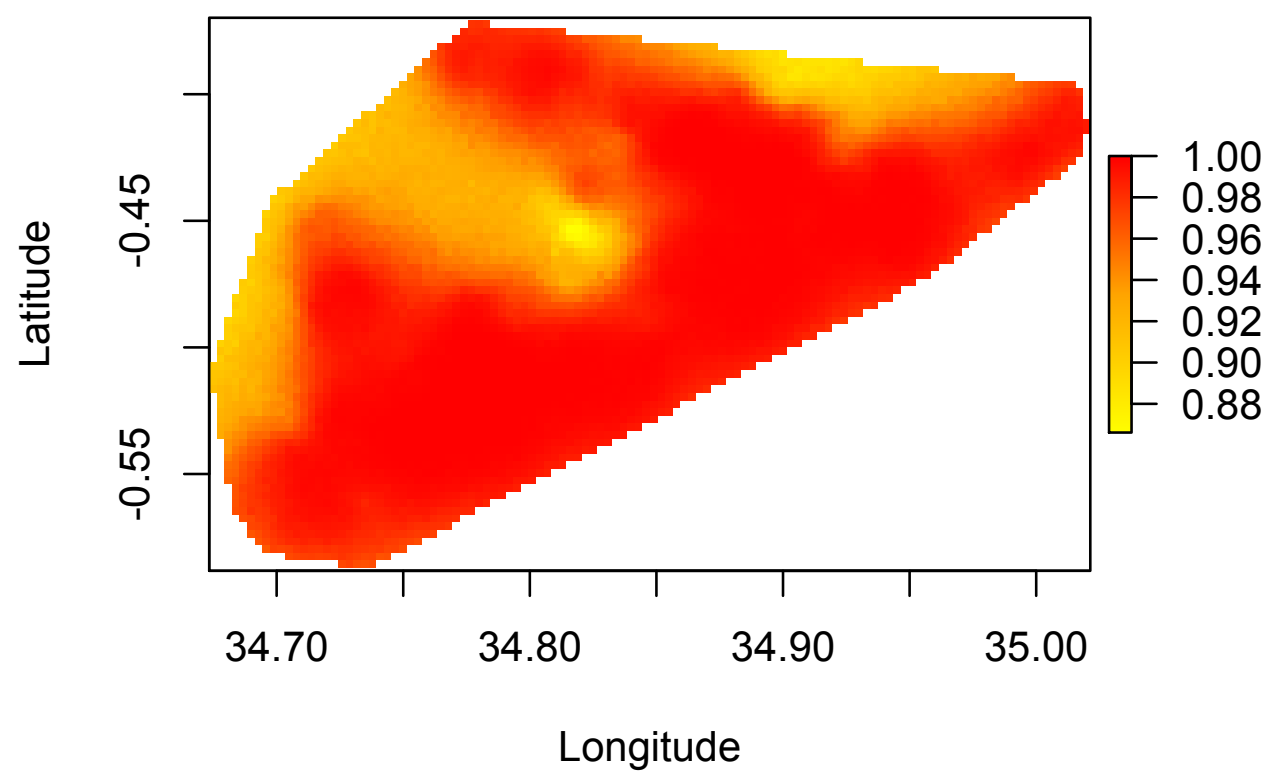

Figure 3: The predicted surfaces for $B^{*}(x)$ (a) and $r(x)(\mathrm{b})$. 
(a)

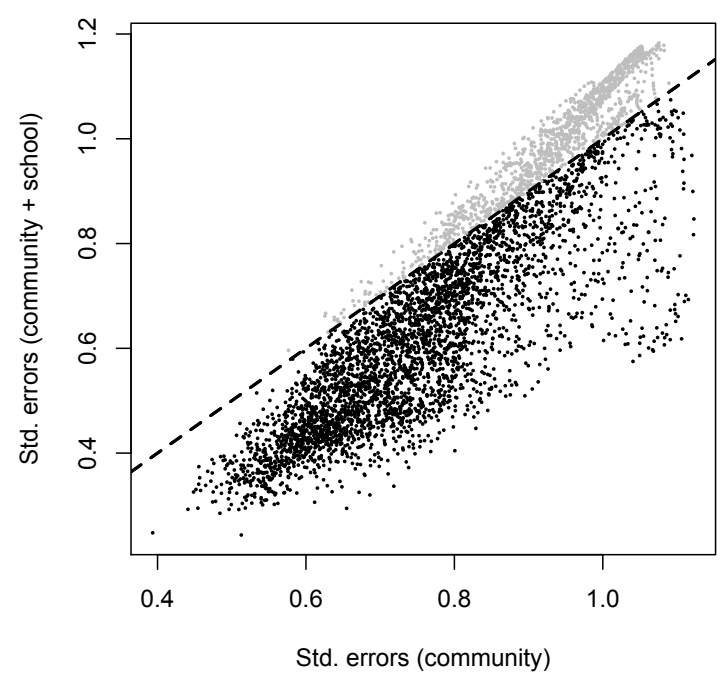

(b)

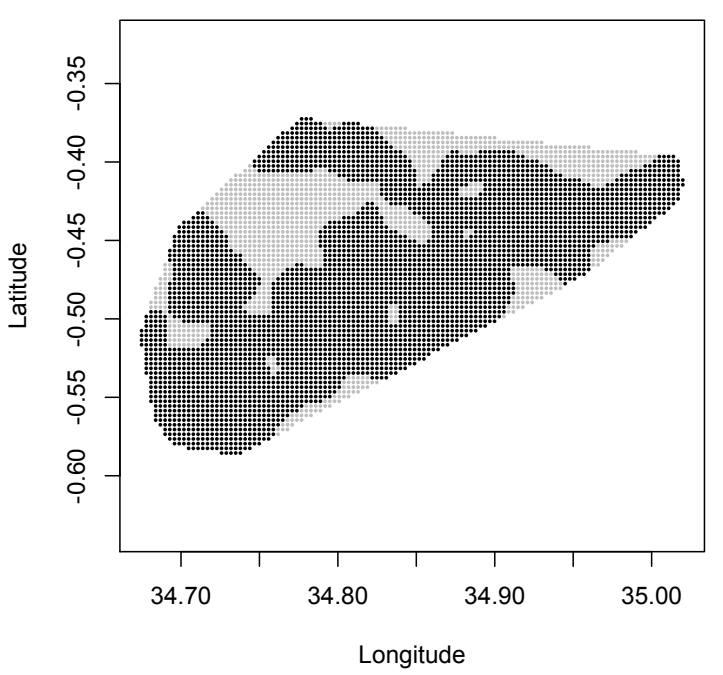

Figure 4: (a) Scatterplot of the standard errors for $S(x)$ using data from the community survey only ( $x$-axis) and using both community and school survey data. Points coloured black or grey lie below or above the identity line $y=x$, respectively. (b) Prediction locations, coloured black or grey at locations where the prediction variance for $S(x)$ is smaller or larger, respectively, when using the data from both the community and school surveys. 


\section{Analysing spatio-temporally referenced prevalence sur- veys}

In endemic disease settings where prevalence varies smoothly over time, joint analysis of data from surveys collected at different times can also bring gains in efficiency. The modelling framework in Giorgi et al. (2015) accommodates multiple surveys conducted at different, discrete times. The extension of (7) to $m$ surveys conducted at possibly different times is

$$
\begin{aligned}
\log \left[p_{k}\left(x_{i}\right) /\left\{1-p_{k}\left(x_{i}\right)\right\}\right]= & d\left(x_{i k}\right)^{\prime} \beta+S_{k}\left(x_{i}\right)+Z_{i k}+ \\
& I(k \in \mathcal{B})\left\{d\left(x_{i k}\right)^{\prime} \delta+B_{k}\left(x_{i}\right)\right\}, k=1, \ldots, m
\end{aligned}
$$

where $\mathcal{B}$ denotes the indices of the non-randomised surveys, $\operatorname{Cov}\left\{S_{k}(x), S_{k}^{\prime}\left(x^{\prime}\right)\right\}=\sigma^{2} \alpha_{k k^{\prime}} \rho\left(x, x^{\prime}\right)$ and $\alpha_{k k^{\prime}}=1$ if surveys $k$ and $k^{\prime}$ are taken at the same time, $-1<\alpha_{k k^{\prime}}<1$ otherwise.

A different design for monitoring endemic disease prevalence is the rolling indicator survey (Roca-Feltrer et al., 2012). This consists of sampling members of a target population of individuals or households more or less continuously over time, the order of sampling being randomised. A natural model for the resulting data is a spatio-temporal version of (2),

$$
\log \left[p\left(x_{i}, t_{i}\right) /\left\{1-p\left(x_{i}, t_{i}\right)\right\}\right]=d\left(x_{i}, t_{i}\right)^{\prime} \beta+S\left(x_{i}, t_{i}\right)+Z_{i},
$$

where now $\left(x_{i}, t_{i}\right)$ denotes the location and time of the $i$ th sample member. There is an extensive literature on ways of specifying the covariance structure of a spatio-temporal Gaussian process; see, for example, Gneiting \& Guttorp (2010). For endemic diseases, a reasonable working assumption is that the relative risk of disease at different times is the same at all locations, and vice versa. This implies an additive formulation,

$$
S(x, t)=S(x)+U(t)
$$


where $S(x)$ and $U(t)$ are independent spatial and temporal Gaussian processes, respectively.

\subsection{Application: rolling malaria indicator survey in Chikwawa dis- trict, Malawi, May 2010 to June 2013}

We now analyse data from a rolling malaria indicator survey (rMIS) conducted in Chikwawa

District, Southern Malawi, from May 2010 to June 2013. In this rMIS, children under five years were randomly selected in 50 villages covering an area of approximately $400 \mathrm{~km}^{2}$. Blood samples were then collected and tested by RDT for malaria. The objectives of the analysis are the following.

(i) interpolation of the spatio-temporal pattern of malaria prevalence for children under twelve months;

(i) estimation of the reduction in prevalence and number of infected children through a scale-up in the distribution of insecticide treated nets (ITN) and delivery of indoor residual spraying (IRS), from the actual coverage to $100 \%$ coverage in each village.

A practical distinction between these two objectives is that the first can only use explanatory variables that are available throughout the study-region, whereas the second can additionally use explanatory variables associated with the sampled households.

\subsubsection{Spatio-temporal interpolation of malaria prevalence}

Let $p_{j}\left(x_{i}, t_{i}\right)$ denote the probability of having a positive RDT outcome for the $j$-th children in the $i$-th household at time $t_{i}$. Using the model defined by (10), the linear predictor assumes 
the form

$$
\begin{aligned}
\log \left[p_{j}\left(x_{i}, t_{i}\right) /\left\{1-p_{j}\left(x_{i}, t_{i}\right)\right\}\right]= & \beta_{0}+\beta_{1} d_{i j}+\beta_{2} t_{i}+\beta_{3} \cos \left(2 \pi t_{i} / 12\right)+\beta_{4} \sin \left(2 \pi t_{i} / 12\right)+ \\
& \beta_{5} \cos \left(2 \pi t_{i} / 6\right)+\beta_{6} \sin \left(2 \pi t_{i} / 6\right)+S\left(x_{i}\right)+U\left(t_{i}\right), \quad
\end{aligned}
$$

where $d_{i j}$ is a binary indicator that takes value 1 if the child is under twelve months and 0 otherwise. The linear combination of sine and cosine functions with periodicities of one year and six months is used to model the seasonality of malaria. For both $S(x)$ and $U(t)$, we use isotropic exponential correlation functions with scale parameters $\phi$ and $\psi$, respectively. We use $\sigma^{2}$ and $\nu^{2} \sigma^{2}$ to denote the variance of $S(x)$ and $U(t)$, respectively.

Table 3 (Model 1) reports the MCML estimates of the model parameters; for the positivevalued parameters $\sigma^{2}, \phi, \nu^{2}$ and $\psi$ we applied a log-transformation to improve the quadratic approximation to the log-likelihood. For the two sinusoidal curves, we also report their associated amplitude, $\sqrt{\beta_{i}^{2}+\beta_{i+1}^{2}}$, and phase, $\tan ^{-1}\left(\beta_{i} / \beta_{i+1}\right)$, where $i=3$ and $i=5$ correspond to periodicities 12 and 6 months, respectively. As expected, the estimate of $\beta_{1}$ indicates a significantly lower risk of having a positive RDT outcome for children in the first year of life, as newborns benefit from maternally acquired immunity that gradually fades.

We now generate prevalence predictions for four of the 50 villages in Chikwawa District. We chose these four villages selectively to include areas of low and high risk for malaria. Let $A_{i}$ denote the convex hull obtained from the sampled locations of the $i$-th village. For a fixed time $t$, we computed

$$
p_{i}(t)=\left|A_{i}\right|^{-1} \int_{A_{i}} \hat{p}(x, t) d x, \text { for } i=1, \ldots, 5
$$

where $d_{i j}$ is fixed at 1 for all $x \in A_{i}$ and $t=1,2, \ldots, 38$, where each integer identifies a month, from May 2010 to June 2013. Also, $\hat{p}(x, t)$ is the mean of the predictive distribution of prevalence at location $x$ and month $t$. For each village, $i$, we approximated the intractable 
Table 3: Monte Carlo Maximum Likelihood estimates for spatio-temporal models fitted to the Malawi malaria prevalence data. Model 1 is defined at equation(12). Model 2 includes three additional explanatory variables: ITN, IRS and SES.

\begin{tabular}{|c|c|c|c|c|}
\hline & \multicolumn{2}{|r|}{ Model 1} & \multicolumn{2}{|r|}{ Model 2} \\
\hline Term & Estimate & 95\% Confidence interval & Estimate & 95\% Confidence interval \\
\hline $\bar{\beta}$ & 4.210 & $(3.815,4.605)$ & 4.644 & $(4.099,5.189)$ \\
\hline$\beta_{1}$ & -5.380 & $(-5.914,-4.847)$ & -5.428 & $(-6.066,-4.789)$ \\
\hline$\beta_{2}$ & -0.067 & $(-0.083,-0.051)$ & -0.072 & $(-0.090,-0.054)$ \\
\hline$\beta_{3}$ & -0.749 & $(-0.978,-0.521)$ & -0.693 & $(-0.922,-0.465)$ \\
\hline$\beta_{4}$ & 0.361 & $(0.134,0.588)$ & 0.160 & $(-0.070,0.389)$ \\
\hline$\beta_{5}$ & -0.099 & $(-0.307,0.109)$ & -0.260 & $(-0.475,-0.045)$ \\
\hline$\beta_{6}$ & -0.168 & $(-0.391,0.055)$ & -0.062 & $(-0.286,0.162)$ \\
\hline$\beta_{7}^{*}$ & - & - & -0.188 & $(-0.492,0.117)$ \\
\hline$\beta_{8}^{* *}$ & - & - & -0.181 & $(-0.503,0.141)$ \\
\hline$\beta_{9}^{* * *}$ & - & - & -0.079 & $(-0.505,0.347)$ \\
\hline $\log \left(\sigma^{2}\right)$ & 0.899 & $(-0.011,1.808)$ & 0.971 & $(0.035,1.906)$ \\
\hline $\log (\phi)$ & -3.624 & $(-4.852,-2.397)$ & -4.463 & $(-5.769,-3.157)$ \\
\hline $\log \left(\nu^{2}\right)$ & -3.282 & $(-4.199,-2.365)$ & -3.118 & $(-4.059,-2.177)$ \\
\hline $\log (\psi)$ & 0.882 & $(-0.170,1.934)$ & 1.118 & $(0.017,2.218)$ \\
\hline$\sqrt{\beta_{3}^{2}+\beta_{4}^{2}}$ & 0.832 & $(0.598,1.065)$ & 0.711 & $(0.480,0.943)$ \\
\hline $\tan ^{-1}\left(\beta_{1} / \beta_{2}\right)$ & 2.692 & $(2.012,3.373)$ & 2.915 & $(2.594,3.234)$ \\
\hline$\sqrt{\beta_{5}^{2}+\beta_{6}^{2}}$ & 0.195 & $(0.000,0.424)$ & 0.267 & $(0.047,0.487)$ \\
\hline $\tan ^{-1}\left(\beta_{5} / \beta_{6}\right)$ & -2.104 & $(-3.134,-1.068)$ & -2.908 & $(-3.976,-1.840)$ \\
\hline
\end{tabular}

integral in (13) using a quadrature method based on a regular grid covering the corresponding $A_{i}$. The results are shown in Figure 5 , where each $p_{i}(t)$ is plotted against $t$; a declining trend of RDT prevalence can be seen, with seasonal troughs and peaks around December-January and April-May, respectively.

For any specified policy-relevant prevalence threshold $\tilde{p}$, a quantity of interest is the predictive probability that the estimated prevalence $\hat{p}(x, t)$ exceeds $\tilde{p}$. In Figure 6 , we map the exceedance probabilities in June of each year for $\tilde{p}=0.2$. Two areas of high and low prevalence are clearly identified. The former corresponds approximately to a flooding area where the the presence of local ponds also favours mosquito breeding. 


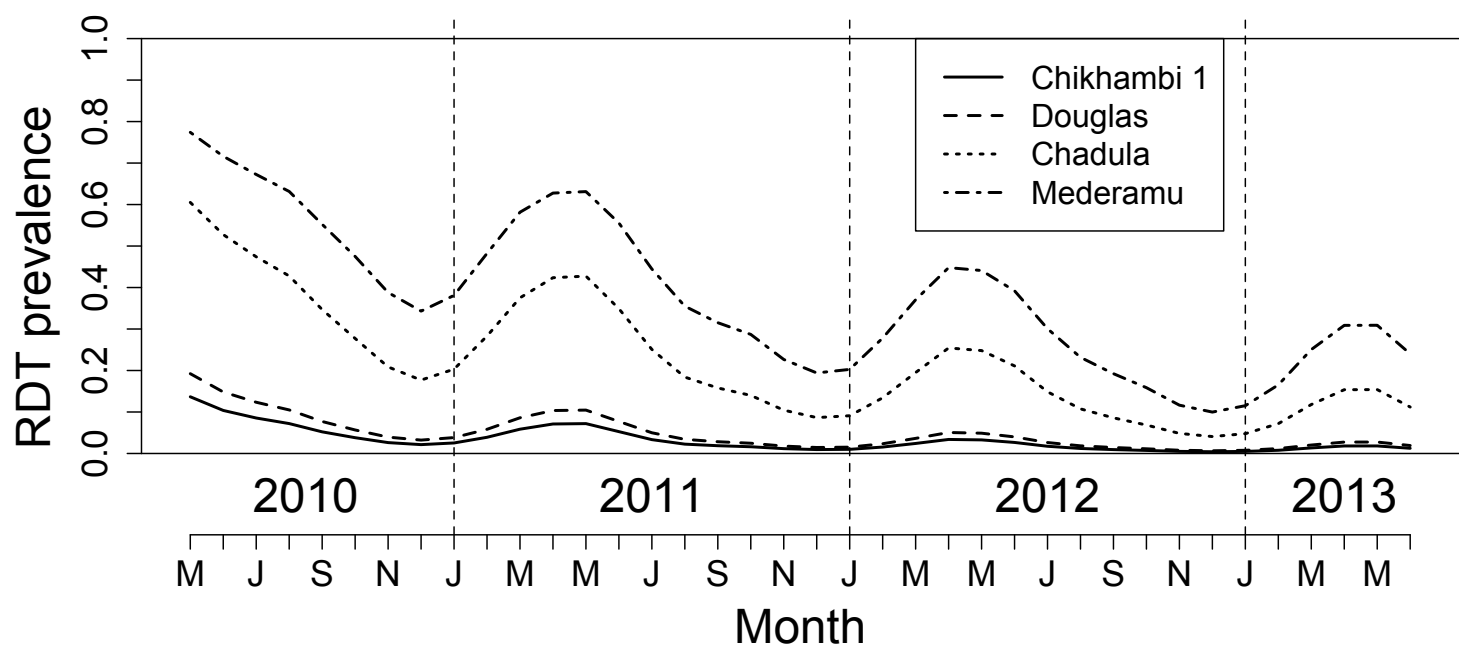

Figure 5: Estimated temporal trend of RDT prevalence for four villages in Chikwawa District. Figure 6 shows the location of each of these five villages.

\subsubsection{Estimating the impact of scaling-up control interventions}

The model (12) that we used to predict malaria prevalence throughout the study-region necessarily excluded any covariate that was only available at the sampled locations. We now propose a procedure to estimate community-wide prevalence and number of infected children under a pre-defined control scenario, focusing on the effects of ownership of ITN and presence of IRS, and adjusting for a measure of each household's socio-economic status (SES, scored from 1 to 5). We first fit a model with linear predictor of the same form in (12), but including these three additional explanatory variables. The resulting parameter estimates are shown in Table 3 (Model 2). We then use enumeration data to obtain, for each village, the total number of children under five years and the number of households with at least one child under five years, and proceed as follows.

(i) Allocate the number of children in each household.

(ii) Impute geographical coordinates, ownership of ITN, presence of IRS and remaining explanatory variables for all unsampled children under the pre-defined control scenario.

(iii) Generate values for all the model parameters using the asymptotic distribution of the 

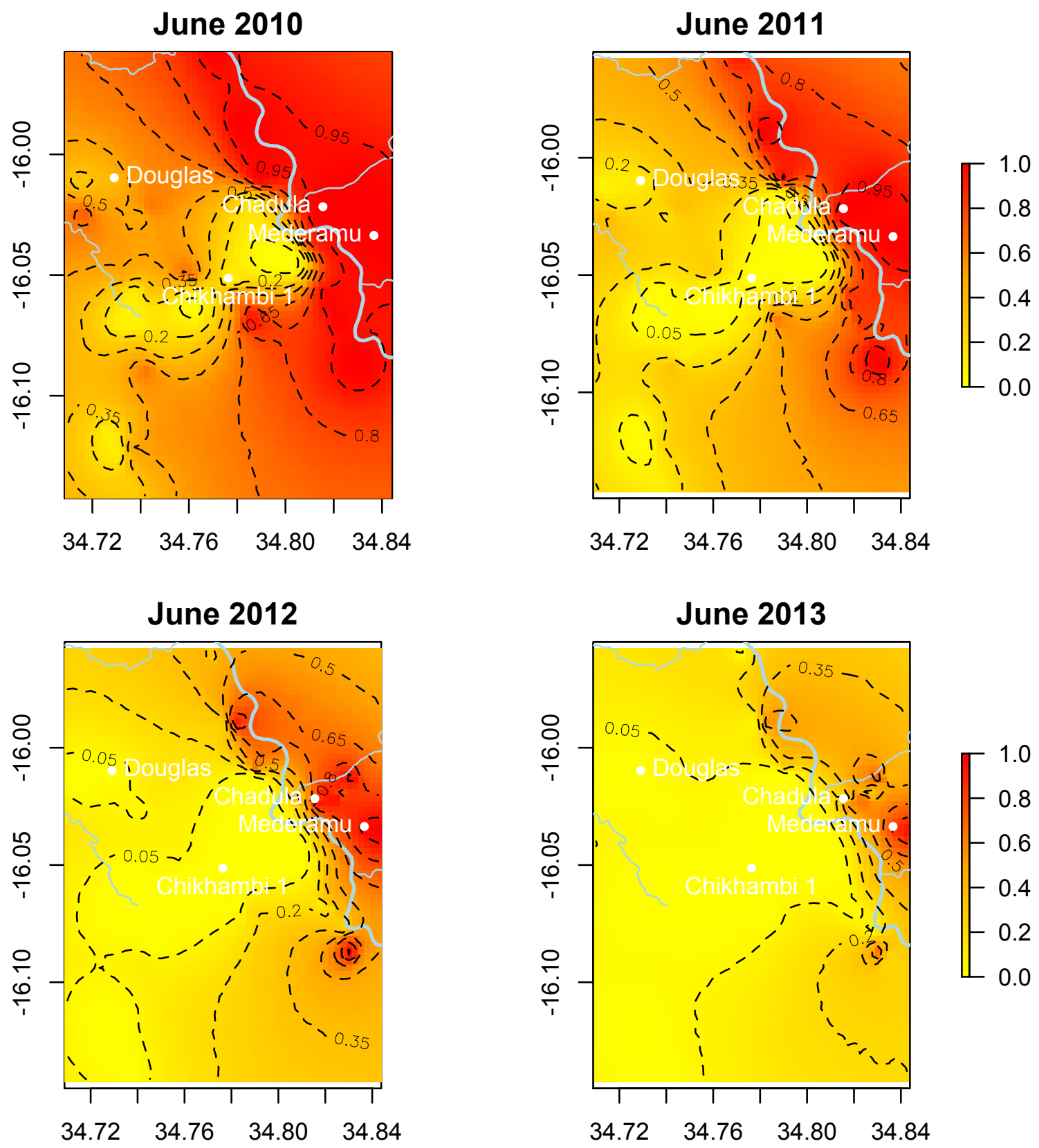

Figure 6: Maps of the predictive exceedance probabilities for a $20 \%$ malaria prevalence threshold in Chikwawa district; light blue lines correspond to waterways, with the Shire river represented by the thicker line. 
maximum likelihood estimator, i.e.

$$
\hat{\theta} \sim N\left(\theta, I_{\text {obs }}^{-1}\right)
$$

where $\theta$ is the vector of model parameters and $I_{\mathrm{obs}}$ is the observed Fisher information as estimated by the negative Hessian of the Monte Carlo likelihood.

(iv) Generate predictive samples for each child's infection status and compute the mean of each sample as a point-estimate of the probability of infection for that child.

(v) For each village, estimate of the number of infected children as the sum of the estimated child-specific probabilities of infection, and average these to estimate the village-level prevalence.

In Step (ii), we imputed the locations of unsampled children by independent random sampling from the uniform distribution over each village area $A_{i}$, defined as the convex hull of the sampled households' locations.

We repeat the above process $N$ times and, for each village, compute summary statistics of the $N$ samples of estimated numbers of infected children and village-level prevalence. We applied this procedure under two different scenarios for April 2013, the most recent peak in RDT prevalence within the period covered by the data, as follows.

S1. Households having IRS and at least one ITN are equally distributed among sampled and unsampled households.

S2. Every household, whether sampled or unsampled, has IRS and at least one ITN.

In scenario S1, we imputed age, ITN, IRS and SES by random sampling from the empirical villlage-level distribution of the sampled households. In scenario S2, only SES and age need 

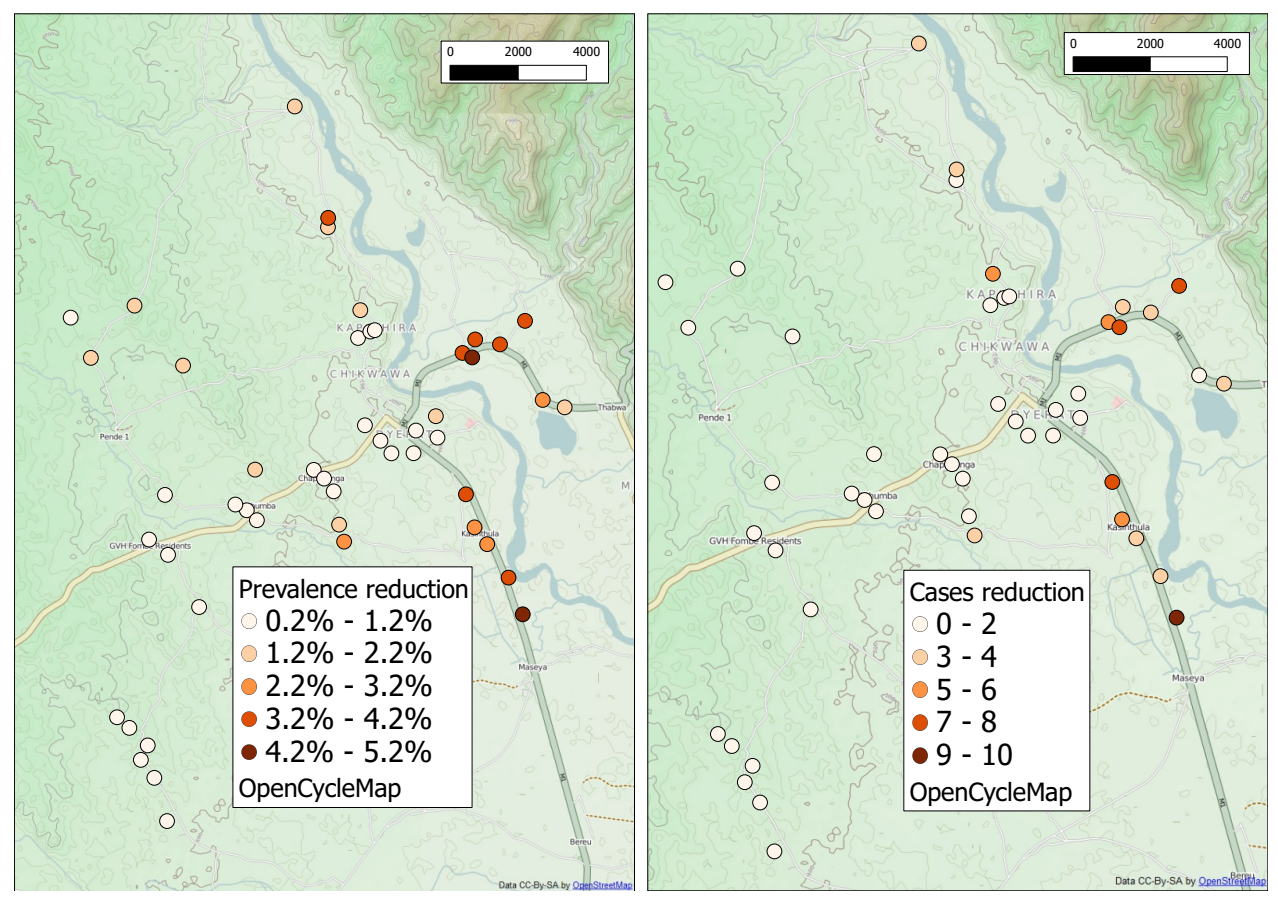

Figure 7: Estimated reduction in prevalence (left panel) and number of infected children (right panel) for each of the 50 villages in Chikwawa District, assuming a scale-up in the distribution of ITN and IRS to $100 \%$ coverage.

to be imputed as ITN and IRS are both present in every household. The differences between estimated prevalences and between numbers of infected children under S2 and S1 are reported in Figure 7. The main gains achieved by scenario S2 are in villages situated in the high prevalence area to the east of the Shire river.

\section{Spatially structured zero-inflation}

The standard geostatistical model for prevalence data in (2) assumes binomial sampling variation around the true prevalence, with a latent risk surface that approaches, but does not exactly reach, zero. However, empirical prevalence data often show an excess of zeros, i.e. zero-inflation. For diseases that are environmentally driven, one explanation for this is that some areas are fundamentally unsuitable for disease transmission. Hence, a zero prevalence estimate in a particular community can be either a chance finding, or a necessary consequence 
of the community being disease/infection-free. Ways of handling spatially structured zeroinflation have been proposed in ecology (Agarwal et al., 2002) and in specific epidemiological applications (Amek et al., 2011; Giardina et al., 2012). These approaches assume that the zero-inflation can be explained by regressing on a limited set of measured risk factors. In this extension to the standard geostatistical model (2) for spatially varying prevalence, $p(x)$, the distribution for the prevalence data $Y$ conditional on $S$ now takes the form of a mixture,

$$
P\left(Y_{i}=y \mid S\left(x_{i}\right)\right)= \begin{cases}{\left[1-\pi\left(x_{i}\right)\right]+\pi\left(x_{i}\right) \operatorname{Bin}\left(0 ; m_{i}, p\left(x_{i}\right)\right)} & \text { if } y=0 \\ \pi\left(x_{i}\right) \operatorname{Bin}\left(y ; m, p\left(x_{i}\right)\right) & \text { if } y>0\end{cases}
$$

where $\pi\left(x_{i}\right) \in(0,1)$ denotes the probability that $x_{i}$ is suitable for transmission of the disease, $\log \left[\pi\left(x_{i}\right) /\left\{1-\pi\left(x_{i}\right)\right\}\right]=d\left(x_{i}\right)^{\prime} \gamma$ and $\operatorname{Bin}(y ; m, p)$ denotes the probability mass function of a binomial distribution with probability of success $p$ and number of trials $m$. The modelled prevalence at location $x$ is $p^{*}(x)=\pi(x) p(x)$.

An alternative way of specifying the conditional distribution of $Y$ given $S$ is given by the so called "hurdle" model (Mullahy, 1986). In this case the mixture distribution for $Y$ assumes the form

$$
P\left(Y_{i}=y \mid S\left(x_{i}\right)\right)=\left\{\begin{array}{ll}
1-\pi\left(x_{i}\right) & \text { if } y=0 \\
\frac{\pi\left(x_{i}\right) \operatorname{Bin}\left(y ; m, p\left(x_{i}\right)\right)}{1-\operatorname{Bin}\left(0 ; m, p\left(x_{i}\right)\right)} & \text { if } y>0
\end{array} .\right.
$$

In our view, (15) is unsuitable for disease mapping for the two following reasons. Firstly, the model does not distinguish between observing no cases amongst sampled individuals as a chance finding or as a necessary consequence of the entire community being disease-free. Secondly, the model can generate unnatural patches of low prevalence around each sampled location for which no cases are observed amongst sampled individuals.

A natural extension of the models in (14) and (15) that allows zero-inflation to depend on both measured and unmeasured covariates can obtained as follows. Define an additional stationary 
Gaussian process $T(x)$ such that

$$
\log \left[\pi\left(x_{i}\right) /\left\{1-\pi\left(x_{i}\right)\right\}\right]=d\left(x_{i}\right)^{\prime} \gamma+T\left(x_{i}\right)
$$

Further decompose the spatial processes $S(x)$ and $T(x)$ as

$$
\begin{aligned}
& S(x)=U_{1}(x)+V(x), \\
& T(x)=U_{2}(x)+V(x)
\end{aligned}
$$

where $U_{1}(x), U_{2}(x)$ and $V(x)$ are independent Gaussian proccesses. In this formulation, $V(x)$ accounts for unmeasured factors that jointly affect the risk of the disease at a location $x$ that is suitable for transmission of the disease and the risk that $x$ is itself suitable for transmission. However, identification of all of the resulting parameters requires a large amount of data. A pragmatic response is to assume that $V(x)=0$ for all $x$, i.e. that $S(x)$ and $T(x)$ are independent processes.

\subsection{Application: river-blindness prevalence mapping}

We now show an application to river-blindness prevalence data, previously analysed in Zouré et al. (2014). Here, we restrict our analysis to three of the twenty APOC countries, namely Mozambique, Malawi and Tanzania. Figure 8 shows the locations of the sampled villages in the three countries. Grey triangles identify the 513 villages with no cases of river-blindness amongst sampled individuals, black dots the 397 villages with at least one case.

We fit the model with conditional distribution for $Y$ given by (14), and logistic link functions (2) and (16) for $p(x)$ and $\pi(x)$, respectively. We also assume that $S(x)$ and $T(x)$ are independent processes with covariance functions $\sigma^{2} \exp (-u / \phi)$ and $\sigma^{2} \omega^{2} \exp (-u / \psi)$, respectively; we denote the variance of the nugget effect $Z$ by $\sigma^{2} \nu^{2}$. We do include covariates, but simply fit constant means $\mu_{1}$ and $\mu_{2}$ on the logit-scale of $p(x)$ and $\pi(x)$, respectively. 


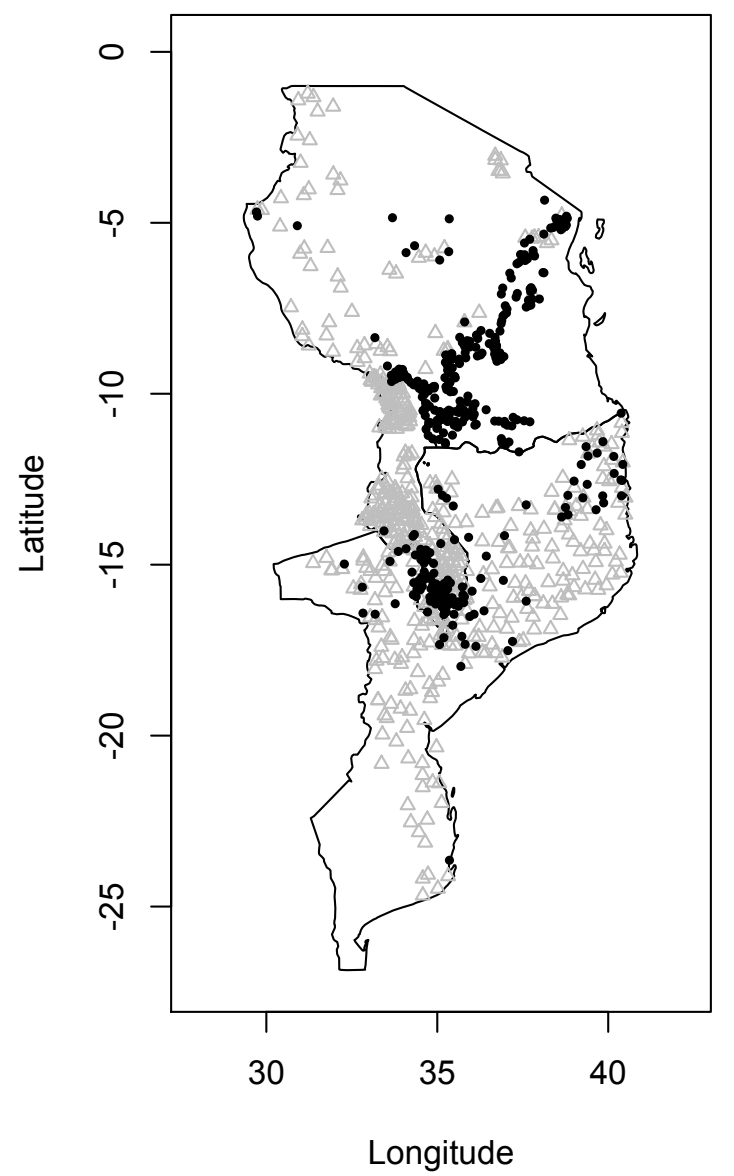

Figure 8: Sampled villages in Mozambique, Malawi and Tanzania, with grey triangles and black dots corresponding to villages with no observed case and at least one observed case of river-blindness, respectively.

Table 4: MCML estimates of parametetrs in the zero-inflated geostatistical model and associated $95 \%$ confidence intervals.

\begin{tabular}{rrc}
\hline Term & Estimate & $95 \%$ confidence interval \\
\hline$\mu_{1}$ & -5.812 & $(-8.746,-2.877)$ \\
$\mu_{2}$ & 2.287 & $(1.361,3.213)$ \\
$\log \left(\sigma^{2}\right)$ & -3.138 & $(-4.075,-2.200)$ \\
$\log \left(\nu^{2}\right)$ & 1.579 & $(0.615,2.543)$ \\
$\log (\phi)$ & -2.899 & $(-6.162,0.363)$ \\
$\log \left(\omega^{2}\right)$ & 2.390 & $(1.425,3.354)$ \\
$\log (\psi)$ & 1.679 & $(0.704,2.654)$ \\
\hline
\end{tabular}


(a)

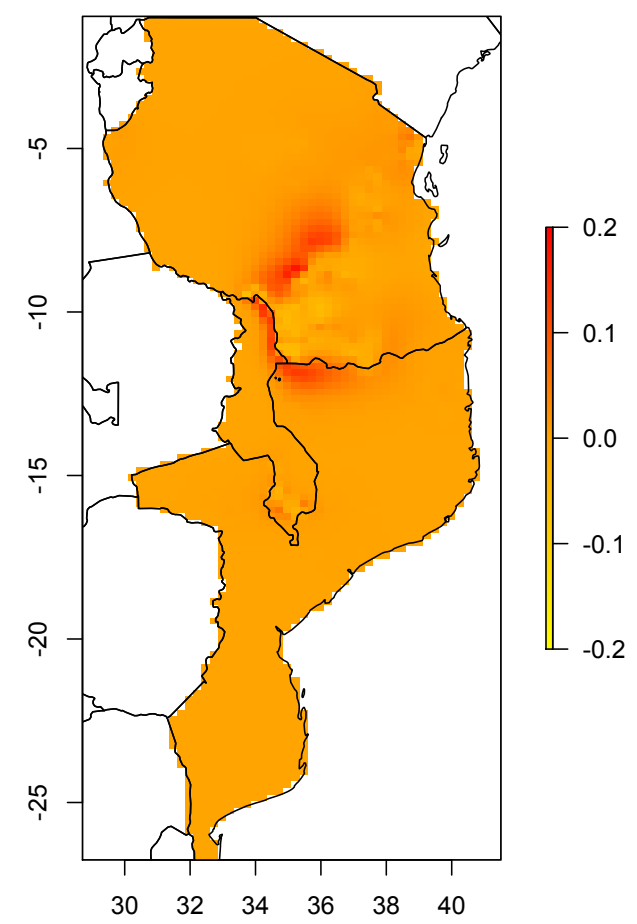

(b)

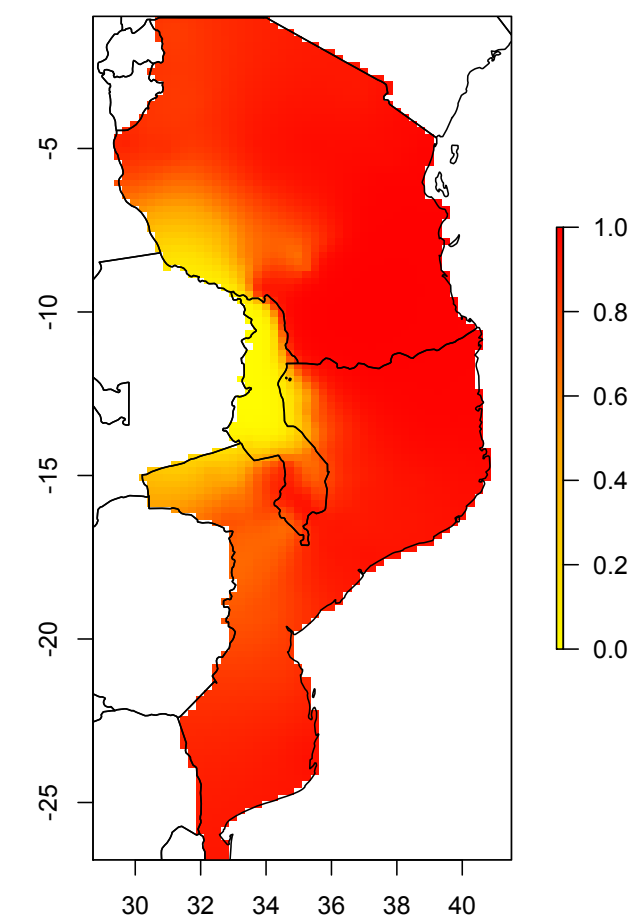

Figure 9: (a) Difference between predicted prevalences using the standard and zero-inflated geostatistical models. (b) Predicted surface $\pi(x)$. 
Table 4 shows the MCML estimates of the model parameters. The estimated scale of the spatial correlation of $T(x)$ is much larger than that of $S(x)$. Also, the estimate of the noiseto-signal ratio $\nu^{2}$ is substantial.

Figure 9(a) shows the difference between estimates of prevalence $\hat{p}_{s}(x)$ and $\hat{p}_{z}(x)$ based on the standard and zero-inflated, geostatistical models, respectively; these range between plus and minus 0.2. Figure $9(\mathrm{~b})$ shows the estimated surface of $\pi(x)$, and indicates that the central and northern parts of Malawi are disease-free, whereas most of the reported zero cases in Mozambique and Tanzania are more likely to be attributable to binomial sampling error.

\section{Discussion}

We have discussed four important issues that arise in prevalence mapping of tropical diseases, namely: combining data from multiple surveys of different quality; spatio-temporal interpolation of disease prevalence; assessment of the impact of control interventions; and accounting for zero-inflation in empirical prevalences. For each issue we have presented an extension of the standard geostatistical model and have described an application that we have encountered through our involvement with public health programmes in Africa.

In all of our applications, we used the exponential correlation function for the process $\mathcal{S}$. As discussed in Section 2, this corresponds to the special case of a Matérn correlation function with smoothness parameter $\kappa=1 / 2$. We did not find evidence against this assumption in any of our applications. Additionally, in our experience, different choices for $\kappa$ lead to only small changes in estimated regression coefficients and the predicted prevalence surface. One reason for this is that prevalence data are inherently imprecise owing to the inescapable element of binomial sampling variation. In other application areas, where precise measurement data are available, the choice between continuous non-differentiable $(\kappa \leq 1)$ and differentiable $(\kappa>1)$ members of the Matérn family may be more important. Our strategy of choosing between a 
few values of $\kappa$, say $\kappa=0.5,1.5$ or 2.5 , is a pragmatic approach acknowledging that $\kappa$ is both poorly identified and typically of limited scientific interest.

As outlined in Section 3, MCML is a useful and feasible computational technique that can be used to fit a very broad class of generalized mixed models. Compared to techniques based on analytical approximations of the likelihood function, such as the Laplace approximation (LA), the MCML method is computationally more intensive. However, subject to computationaly feasibility, it is more accurate and, especially in the case of small binomial denominators, can provide more reliable inferences (Joe, 2008).

In the applications of Section 4.1 and Section 5.1, we considered extra-binomial variation at household-level but not at individual-level within households. An extension of the standard geostatistical model (2) that accounts for within-household random variation is

$$
\log \left\{p_{i j} /\left(1-p_{i j}\right)\right\}=\alpha+\left[c_{i j}^{\prime} \delta+U_{i j}\right]+\left[d\left(x_{i}\right)^{\prime} \beta+S\left(x_{i}\right)+Z_{i}\right]
$$

where $i$ denotes household, $j$ denotes individual within household, $c_{i j}$ is a vector of individualspecific explanatory variables with associated regression parameters $\delta$ and the $V_{i j}$ are mutually independent, zero-mean, Normally distributed random effects. However, when the data consists of empirical prevalences with small denominators, it is generally difficult to disentangle the effects of $Z_{i}$ and $U_{i j}$. For this reason we used the more pragmatic approach of setting $U_{i j}=0$ for all $i$ and $j$.

The results of Section 5.1.2 on the impact of scaling-up the distribution of ITN and IRS to $100 \%$ coverage should be interpreted cautiously. The procedure that we used to obtain estimates of prevalence and number of infected children under different scenarios does not deal with the issue of causation. The control scenarios S1 and S2 represent virtual scenarios under which coverage of ITN and IRS is assumed to follow a pre-defined pattern without having any impact on other risk factors for malaria. In reality, a scale-up of ITN and IRS coverage may influence other features of the process, for example the extent to which ITNs 
are used correctly.

Under model (14) that accounts for zero-inflation, the risk surface can approach, but not reach, zero. We are are currently working on two further extensions of the standard geostatistical model. In the first of these, prevalence can reach zero but is constrained to do so smoothly. The second allows discontinuities in risk between suitable and unsuitable areas of transmission. Spatial discontinuities may seem artificial but can give a better fit to the data, especially when the pattern of risk is highly non-linear. Statistical tools for automatic choice between non-nested models are available from both frequentist and Bayesian perspectives, but our preference is to reach agreement with a subject-matter expert on what qualitative features of the model best reflect the behaviour of the underlying process.

\section{Acknowledgements}

We thank the following people for providing the data analysed in the paper and helpful discussions: Dr. Gillian Stresman and Dr. Jennifer Stevenson (community and school-children data); Dr. Anja Terlouw (rMIS data); Dr. Hans Remme (river blindness data).

Support was provided by the award of a UK Economic and Social Research Council PhD studentship (ESRC Grant No.: ES/J500094/1) to Emanuele Giorgi and by The Farr Institute@HeRC. The Farr Institute@HeRC is supported by a 10-funder consortium: Arthritis Research UK, the British Heart Foundation, Cancer Research UK, the Economic and Social Research Council, the Engineering and Physical Sciences Research Council, the Medical Research Council, the National Institute of Health Research, the National Institute for Social Care and Health Research (Welsh Assembly Government), the Chief Scientist Office (Scottish Government Health Directorates) and the Wellcome Trust (MRC Grant No.: MR/K006665/1). 


\section{References}

Agarwal, D. K., Gelfand, A. E. \& Citron-Pousty, S. (2002). Zero-inflated models with application to spatial count data. Environmental and Ecological Statistics 9, 341-355.

Amek, N., Bayoh, N., Hamel, M., Lindblade, K. A., Gimnig, J., Laserson, K. F., Slutsker, L., Smith, T. \& Vounatsou, P. (2011). Spatio-temporal modeling of sparse geostatistical malaria sporozoite rate data using a zero inflated binomial model. Spatial and Spatio-temporal Epidemiology 2, 283-290.

Besag, J. \& Mondal, D. (2005). First-order intrinsic autoregressions and the de Wijs process. Biometrika 92, 909-920.

Chilés, J.-P. \& Delfiner, P. (2012). Geostatistics: Modelling Spatial Uncertainty. Wiley, New York, 2nd ed.

Claridge, J., Diggle, P. J., McCann, C. M., Mulcahy, G., Flynn, R., McNair, J., Strain, S., Welsh, M., Baylis, M. \& Williams, D. J. L. (2012). Fasciola hepatica is associated with the failure to detect bovine tuberculosis in dairy cattle. Nature Communications 3, 853.

Clements, A., Lwambo, N., Blair, L., Nyandindi, U., Kaatano, G., Kinung'hi, S., Webster, J., Fenwick, A. \& Brooker, S. (2006). Bayesian spatial analysis and disease mapping: tools to enhance planning and implementation of a schistosomiasis control programme in tanzania. Tropical Medicine and International Health 11, 490-503.

Cox, D. R. (1955). Some statistical methods connected with series of events. Journal of the Royal Statistical Society, Series B 17, 129-164.

Cressie, N. (1993). Statistics for spatial data. Wiley, New York.

De WiJs, H. J. (1951). Statistics of ore distribution. Part I. Frequency distributions of assay values. Journal of the Royal Netherlands Geological and Mining Society 13, 365-375. 
De WiJs, H. J. (1953). Statistics of ore distribution. Part II. Theory of binomial distributions applied to sampling and engineering problems. Journal of the Royal Netherlands Geological and Mining Society 15, 12-24.

Diggle, P., Thomson, M., Christensen, O., Rowlingson, B., Obsomer, V., Gardon, J., Wanji, S., Takougang, I., Enyong, P., Kamgno, J., Remme, J., BoussiNESQ, M. \& Molyneux, D. (2007). Spatial modelling and the prediction of loa loa risk: decision making under uncertainty. Annals of Tropical Medicine and Parasitology 101, 499-509.

Diggle, P. J., Moraga, P., Rowlingson, B. \& Taylor, B. M. (2013). Spatial and spatio-temporal log-Gaussian Cox processes: Extending the geostatistical paradigm. Statistical Science 28, 542-563.

Diggle, P. J., Moyeed, R., Rowlingson, B. \& Thomson, M. (2002). Childhood malaria in the Gambia: a case-study in model-based geostatistics. Journal of the Royal Statistical Society, Series C 51, 493-506.

Diggle, P. J., Tawn, J. A. \& Moyeed, R. A. (1998). Model-based geostatistics (with discussion). Applied Statistics 47, 299-350.

Gemperli, A., Vounatsou, P., Kleinschmidt, I., Bagayoko, M., Lengeler, C. \& Smith, T. (2004). Spatial patterns of infant mortality in Mali: The effect of malaria endemicity. American Journal of Epidemiology 159, 64-72.

Gething, P. W., Elyazar, I. R. F., Moyes, C. L., Smith, D. L., Battle, K. E., Guerra, C. A., Patil, A. P., Tatem, A. J., Howes, R. E., Myers, M. F., George, D. B., Horby, P., Wertheim, H. F. L., Price, R. N., Meller, I., Baird, J. K. \& HAY, S. I. (2012). A long neglected world malaria map: Plasmodium vivax endemicity in 2010. PLoS Neglected Tropical Diseases 6, e1814. 
Giardina, F., Gosoniu, L., Konate, L., Diouf, M. B., Perry, R., Gaye, O., Faye, O. \& Vounatsou, P. (2012). Estimating the burden of malaria in Senegal: Bayesian zero-inflated binomial geostatistical modeling of the MIS 2008 data. PLoS ONE 7, e32625.

Giorgi, E. \& Diggle, P. J. (2014). Prevmap: an R package for prevalence mapping. Submitted.

Giorgi, E., Sesay, S. S. S., Terlouw, D. J. \& Diggle, P. J. (2015). Combining data from multiple spatially referenced prevalence surveys using generalized linear geostatistical models. Journal of the Royal Statistical Society, Series A 178, 445-464.

Gneiting, T. \& Guttorp, P. (2010). Continuous parameter spatio-temporal processes. In Handbook of Spatial Statistics, A. E. Gelfand, P. J. Diggle, M. Fuentes \& P. Guttorp, eds. Boca Raton: Chapman and Hall/CRC Press, pp. 427-436.

Gotway, C. A. \& Stroup, W. W. (1997). A generalized linear model approach to spatial data analysis and prediction. Journal of Agricultural, Biological, and Environmental Statistics 2, 157-178.

Hay, S. I., Guerra, C. A., Gething, P. W., Patil, A. P., Tatem, A. J., Noor, A. M., Kabaria, C. W., Manh, B. H., Elyazar, I. R. F., Brooker, S., Smith, D. L., Moyeed, R. A. \& Snow, R. W. (2009). A world malaria map: Plasmodium falciparum endemicity in 2007. PLoS Medicine 6, e1000048.

Hedt, B. L. \& Pagano, M. (2011). Health indicators: Eliminating bias from convenience sampling estimator. Statistics in Medicine 30, 560-568.

Higdon, D. (1998). A process-convolution approach to modeling temperatures in the North Atlantic Ocean. Environmental and Ecological Statistics 5, 173-190.

Higdon, D. (2002). Space and space-time modeling using process convolutions. In Quantita- 
tive methods for current environmental issues, C. W. Anderson, V. Barnett, P. C. Chatwin \& A. H. El-Shaarawi, eds. Springer-Verlag, New York, pp. 37-56.

Joe, H. (2008). Accuracy of laplace approximation for discrete response mixed models. Computational Statistics \& Data Analysis 52, 5066 - 5074.

Kleinschmidt, I., Pettifor, A., Morris, N., MacPhail, C. \& Rees, H. (2007). Geographic distribution of human immunodeficiency virus in South Africa. The American journal of tropical medicine and hygiene 77, 1163-1169.

Kleinschmidt, I., Sharp, B. L., Clarke, G. P. Y., Curtis, B. \& Fraser, C. (2001). Use of generalized linear mixed models in the spatial analysis of small-area malaria incidence rates in Kwazulu Natal, South Africa. American Journal of Epidemiology 153, 1213-1221.

Krige, D. G. (1951). A statistical approach to some basiv mine valuation problems on the witwatersrand. Journal of the Chemical, Metallurgical and Mining Society of South Africa 52, 119-139.

LiAnG, K. \& ZEGER, S. L. (1986). Longitudinal data analysis using generalized linear models. Biometrika 73, 13-22.

Lindgren, F., Rue, H. \& Lindstrm, J. (2011). An explicit link between gaussian fields and gaussian markov random fields: the stochastic partial differential equation approach. Journal of the Royal Statistical Society: Series B 73, 423-498.

MatÉRn, B. (1986). Spatial Variation. Springer, Berlin, 2nd ed.

Mullahy, J. (1986). Specification and testing of some modified count data models. Journal of Econometrics 33, 341-365.

Pullan, R. L., Gething, P. W., Smith, J. L., Mwandawiro, C. S., Sturrock, H. J. W., Gitonga, C. W., Hay, S. I. \& Brooker, S. (2011). Spatial modelling of soil- 
transmitted helminth infections in Kenya: A disease control planning tool. PLoS Neglected Tropical Diseases 5, e958.

Raso, G., Matthys, B., N'Goran, E. K., Tanner, B., Vounatsou, P. \& Utzinger, J. (2005). Spatial risk prediction and mapping of schistosoma mansoni infections among schoolchildren living in western Côte d'Ivoire. Parasitology 131, 97-108.

Roca-Feltrer, A., Lalloo, D. J., Phiri, K. \& Terlouw, D. J. (2012). Rolling malaria indicator surveys (rMIS): A potential district-level malaria monitoring and evaluation (M \& E) tool for program managers. American Journal of Tropical Medicine and Hygiene 86, 96-98.

Rue, H., Martino, S. \& Chopin, N. (2009). Approximate Bayesian inference for latent Gaussian models by using integrated nested laplace approximations. Journal of the Royal Statistical Society, Series B 71, 319-392.

Soares Magalhaes, R. J. \& Clements, A. C. A. (2011). Mapping the risk of anaemia in preschool-age children: The contribution of malnutrition, malaria, and helminth infections in West Africa. PLoS Medicine 8, e1000438.

Stein, M. (1999). Interpolation of Spatial Data: Some Theory for Kriging. Springer, New York.

Stevenson, J. C., Stresman, G. H., Gitonga, C. W., Gillig, J., Owaga, C., Marube, E., Odongo, W., Okoth, A., China, P., Oriango, R., Brooker, S. J., Bousema, T., Drakeley, C. \& Cox, J. (2013). Reliability of school surveys in estimating geographic variation in malaria transmission in the Western Kenyan highlands. PLoS ONE 8, e77641.

Thomson, M., Obsomer, V., Kamgno, J., Gardon, J., Wanji, S., Takougang, I., Enyong, P., Remme, J., Molyneux, D. \& Boussinesq, M. (2004). Mapping the 
distribution of Loa loa in Cameroon in support of the African Programme for Onchocerciasis Control. Filaria Journal 3, 7. DOI:10.1186/1475-2883-3-7.

WHO (2012). African Programme for Onchocerciasis Control: meeting of national onchocerciasis task forces. Weekly epidemiological record 87, 493-508.

ZHANG, H. (2004). Inconsistent estimation and asymptotically equal interpolations in modelbased geostatistics. Journal of the American Statistical Association 99, 250-261.

Zouré, Honorat, G. M., Noma, M., Tekle, Afework, H., Amazigo, U. V., Diggle, P. J., Giorgi, E. \& Remme, J. H. F. (2014). The geographic distribution of onchocerciasis in the 20 participating countries of the african programme for onchocerciasis control: (2) pre-control endemicity levels and estimated number infected. Parasites \& Vectors 7. 